\title{
Human telomerase catalytic subunit (hTERT) suppresses p53-mediated anti-apoptotic response via induction of basic fibroblast growth factor
}

\author{
Xun Jin ${ }^{1 *}$, Samuel Beck ${ }^{2 *}$, Young-Woo Sohn ${ }^{1}$ \\ Jun-Kyum Kim ${ }^{1}$, Sung-Hak Kim ${ }^{1}$, Jinlong Yin ${ }^{2}$, \\ Xumin Pian', Sung-Chan Kim ${ }^{3}$, Yun-Jaie Choi ${ }^{2,4}$ \\ and Hyunggee $\mathrm{Kim}^{1,4}$ \\ ${ }^{1}$ Cell Growth Regulation Laboratory (CGRL) \\ School of Life Sciences and Biotechnology \\ Korea University \\ Seoul 136-713, Korea \\ ${ }^{2}$ National Research Laboratory of Animal Cell Biotechnology \\ School of Agricultural Biotechnology \\ Seoul National University \\ Seoul 152-742, Korea \\ ${ }^{3}$ Department of Biochemistry \\ College of Medicine, Hallym University \\ Chuncheon 200-702, Korea \\ ${ }^{4}$ Corresponding authors: Tel, 82-2-3290-3059; \\ Fax, 82-2-3290-3475; E-mail, hg-kim@korea.ac.kr (H.G.K.) \\ Tel, 82-2-880-4816; FAX, 82-2-880-4807; \\ E-mail: cyjcow@snu.ac.kr (Y.J.C.) \\ *These authors contributed equally to this work. \\ DOI 10.3858/emm.2010.42.8.058
}

Accepted 14 July 2010

Available Online 14 July 2010

Abbreviations: bFGF, basic fibroblast growth factor; EGFR, epidermal growth factor receptor; HFFS, human fetal fibroblast

\begin{abstract}
Although human telomerase catalytic subunit (TERT) has several cellular functions including telomere homeostasis, genomic stability, cell proliferation, and tumorigenesis, the molecular mechanism underlying anti-apoptosis regulated by TERT remains to be elucidated. Here, we show that ectopic expression of TERT in spontaneously immortalized human fetal fibroblast (HFFS) cells, which are a telomerase- and p53-positive, leads to increases of cell proliferation and transformation, as well as a resistance to DNA damage response and inactivation of p53 function. We found that TERT and a mutant TERT (no telomerase activity) induce expression of basic fibroblast growth factor (bFGF), and ectopic expression of bFGF also allows cells to be resistant to DNA-damaging response and to
\end{abstract}

suppress activation of p53 function under DNA-damaging induction. Furthermore, loss of TERT or bFGF markedly increases a p53 activity and DNA-damage sensitivity in HFFS, HeLa and U87MG cells. Therefore, our findings indicate that a novel TERT-bFGF axis accelerates the inactivation of p53 and consequent increase of resistance to DNA-damage response.

Keywords: apoptosis; cell death; fibroblast growth factor 2; telomerase; tumor suppressor protein p53

\section{Introduction}

The most spaciously established function of telomerase is to synthesize telomeric repeat (TTAGGG)n onto chromosome ends (telomere) and thus maintains integrity of the telomeres during cell division (Greider, 1996). Telomerase activity is barely detectable in somatic cells, but is reactivated in immortalized cell lines and in $80-90 \%$ of all human cancers (Shay, and Bacchetti,1997; Hiyama and Hiyama, 2002). It has been documented that telomerase activity and expression levels of TERT mRNA are correlated with clinical aggressiveness and relatively differ in various types of tumors (Tomoda et al., 2002; Branca et al., 2007). On the other hand, ectopic expression of TERT is able to induce the resistance to several forms of cell death and the accelerated cell growth (Gorbunova et al., 2002; Luiten et al., 2003; Lee et al., 2005), whereas depletion of TERT by RNA interference results in an enhanced sensitivity to cell death and consequent growth inhibition in cancer cells (Kurvinen et al., 2006; Wang et al., 2007).

Although it is well documented that maintenance of telomere length, which is associated with tumor initiation and tumor cell proliferation, is one of the fundamental roles of telomerase, its other functions independent of telomere elongation have been also suggested that overexpression of telomerase promotes tumorigenesis without changing the telomere length (Rudolph et al., 2001; Papadopoulou et al., 2003; Cayuela et al., 2005). Furthermore, telomerase accelerates cell growth and tumorigenesis through modulating expression levels of a number of genes, such as epidermal growth factor receptor (EGFR), basic fibroblast growth factor 
A

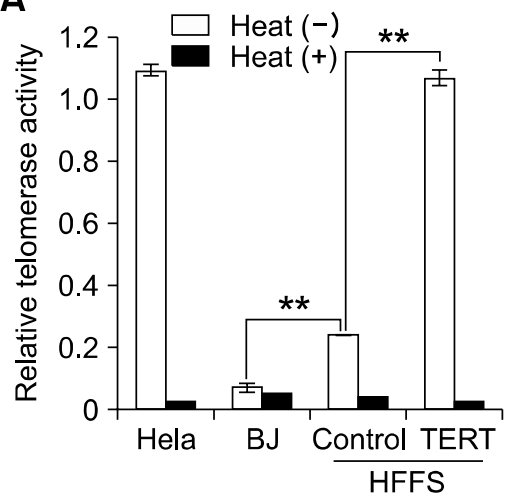

D

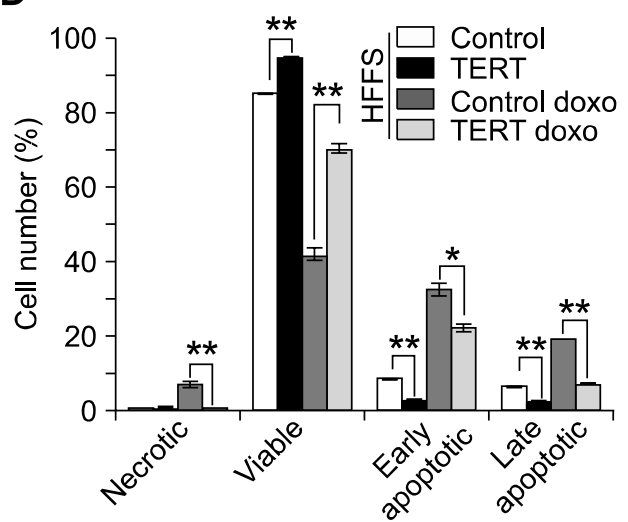

B

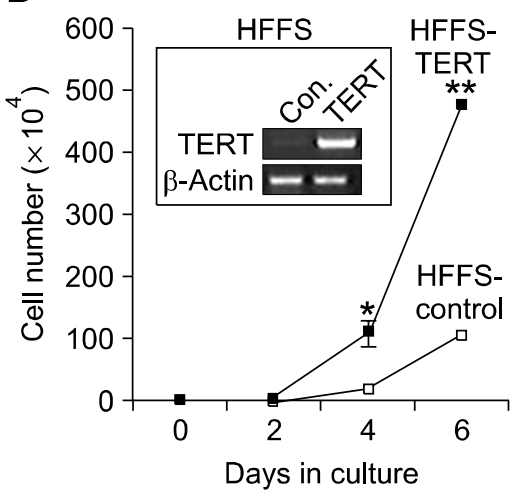

E

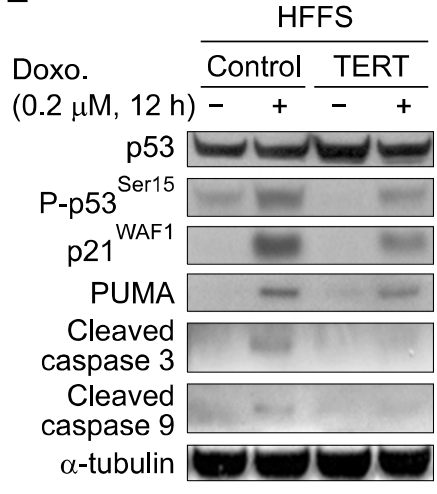

C

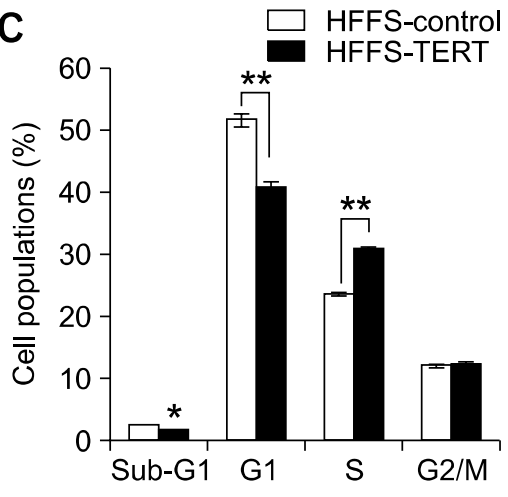

Figure 1. Ectopic expression of TERT in HFFS leads to increase in cell proliferation and decrease in p53-mediated apoptosis. (A) Relative telomerase activities in HeLa, BJ, HFFS-control, and HFFS-TERT cells. HeLa and BJ cells are used for a positive and negative controls for telomerase activity, respectively. Since telomerase is a heat-sensitive enzyme, telomerase activity of each cells was compared by treatment with (heat + ) or without (heat-) heat incubation at $95^{\circ} \mathrm{C}$ for $15 \mathrm{~min}$. (B) Cell proliferation rates of HFFS-control and HFFS-TERT cells. Expression levels of TERT mRNA are determined by semi-quantitative RT-PCR (inset). (C) Cell cycle profiles are determined by propidium iodide (PI)-staining and FACS analysis. (D) Apoptotic cell population of HFFS-control and HFFS-TERT cells grown in the presence and absence of doxorubicin (1 uM, $12 \mathrm{~h})$ are determined by AnnexinV-PI staining and FACS analysis. (E) Expression levels of total p53, phosphorylated p53 (Ser15), p21 ${ }^{\text {WAF1 }}$, PUMA, cleaved caspase 3 and caspase 9 proteins are determined by Western blot analysis. Transcriptional activity of p53 was examined by measuring luciferase activity of p21 ${ }^{\text {WAF1 }}$-promoter- and PUMA-promoter-reporter vectors.

(bFGF), and c-Met oncogenes (Smith et al., 2003). However, the precise mechanism of anti-apoptosis governed by the telomere lengthening-independent activity of telomerase is less elucidated. In the present study, we demonstrated that excessive expression of TERT promotes anti-apoptotic response through induction of bFGF expression and consequent inactivation of p53 tumor suppressor, which is independent of alteration in telomere length.

\section{Results}

\section{Effect of ectopic TERT expression on proliferation and transformation of spontaneously immortalized human fetal fibroblasts}

Although most of cancer cells are shown to have
TERT expression and its functional activity to maintain telomere length, its expression level relatively differs in various types of tumors. To determine biological significance achieved by different expression levels of TERT, we first transduced TERT in spontaneously immortalized human fetal fibroblasts (HFFS) that are telomerase- (Figure 1A) and p53-positive (Figure 1E). We found that telomerase activity was markedly increased in TERT-transduced HFFS (HFFS-TERT) at a similar level to HeLa cells (Figure 1A). Cell morphology of HFFS was not evidently changed by ectopic expression of TERT (data not shown), whereas their proliferation rates were significantly increased as compared to control cells (HFFS-control) (Figure 1B). Furthermore, FACS analysis showed significant increase of $S$ phase, while decrease of G1 phase in the HFFS-TERT cells compared to 
A

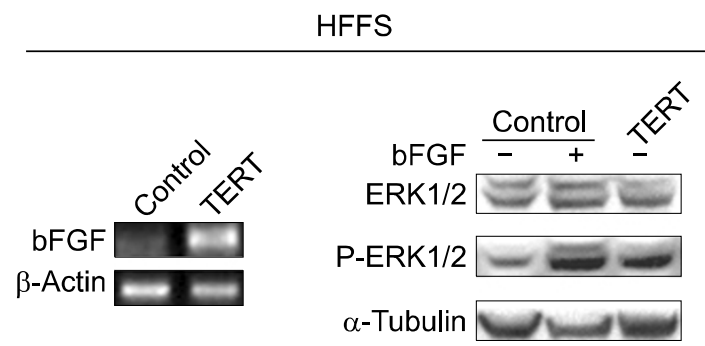

B

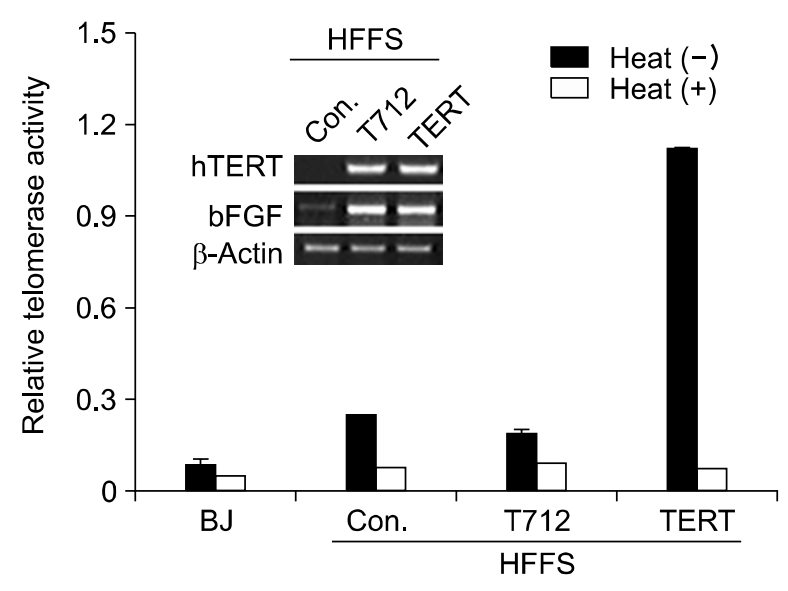

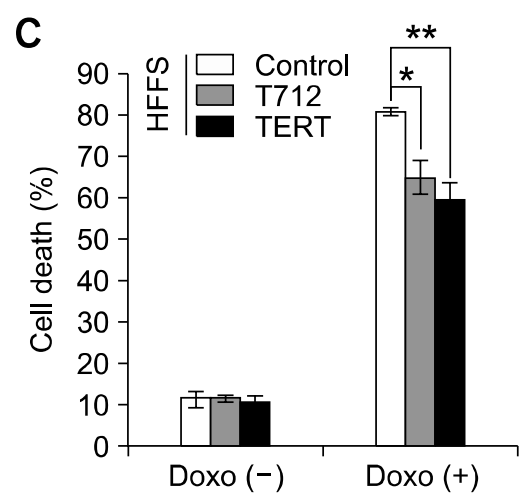
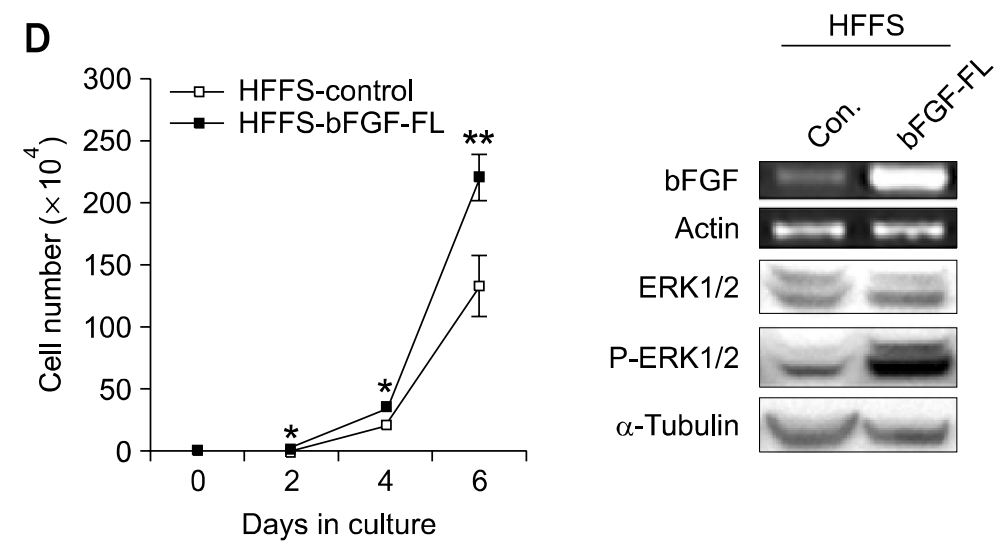

Figure 2. Ectopic expression of TERT induces bFGF expression. (A) bFGF mRNA level is determined by semi-quantitative RT-PCR. Phosphorylated ERK1/2 proteins in the HFFS-TERT cells and bFGF-treated HFFS cells $(20 \mathrm{ng} / \mathrm{ml}, 3 \mathrm{~h})$ are examined by Western blot analysis. (B) Relative telomerase activities of BJ, HFFS-control, HFFS-TERT-D712A (T712), and HFFS-TERT cells. Expression levels of TERT and bFGF mRNAs in these cells are determined by semi-quantitative RT-PCR (inset). (C) Cell death rates are determined by trypan blue staining. Doxorubicin (1 uM, $12 \mathrm{~h}$ ). (D) Cell proliferation rates of HFFS-control and HFFS-bFGF cells (left graph). Expression levels of bFGF mRNA and phosphorylated ERK1/2 proteins in these cells (right panel).

HFFS-control cells (Figure 1C).

\section{Effect of ectopic TERT expression on the DNA damage response and p53 function}

We examined whether ectopic expression of TERT allows cells to be resistant to cytotoxic stimuli, such as treatment of DNA-damaging agents. HFFS-TERT cells grown in the presence of doxorubicin are more resistant to cell death and showed significant decreases in numbers of early and late apoptotic as well as necrotic cells as compared to HFFS-control cells, (Figure 1D and Supplemental Data Figure S1A). Since p53 tumor suppressor activated by DNA-damaging agents regulates anti-proliferative response by controlling the expression of $\mathrm{p} 21^{\mathrm{WAF} 1}$, Puma, Noxa, and Bax
(Aylon et al., 2007), we examined whether the cell death resistance of HFFS-TERT cells to DNA-damaging agents is associated with inactivation of p53 by analyzing the expression levels of its downstream-target genes and its transcriptional activity using promoter-reporter gene assay. We found that expression levels of phosphorylated p53 (Ser15), p21 ${ }^{\text {WAF1 }}$, Puma and cleaved caspase 3 and caspase 9 proteins, as well as p53 transcriptional activity to $\mathrm{p} 21^{\text {WAF } 1}$ and Puma promoters were relatively decreased in HFFS-TERT cells compared to HFFS-control cells, as treated with doxorubicin (Figure 1E). These results indicate that ectopic expression of TERT suppresses activation of $\mathrm{p} 53$ protein in the presence of DNA-damaging stimulus and consequently inhibits apoptotic cell death. 
A

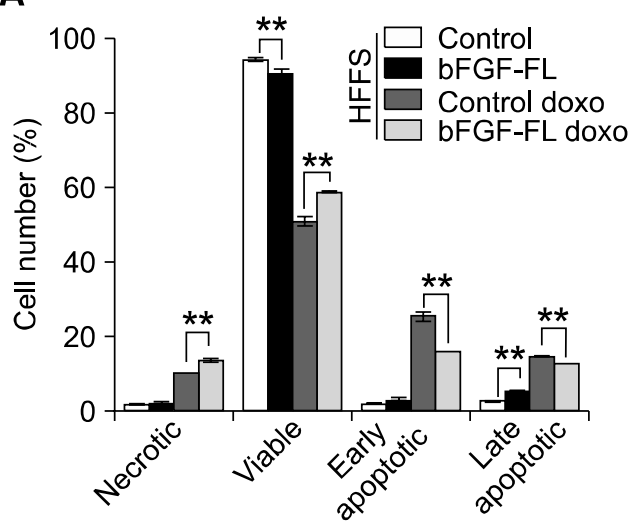

C

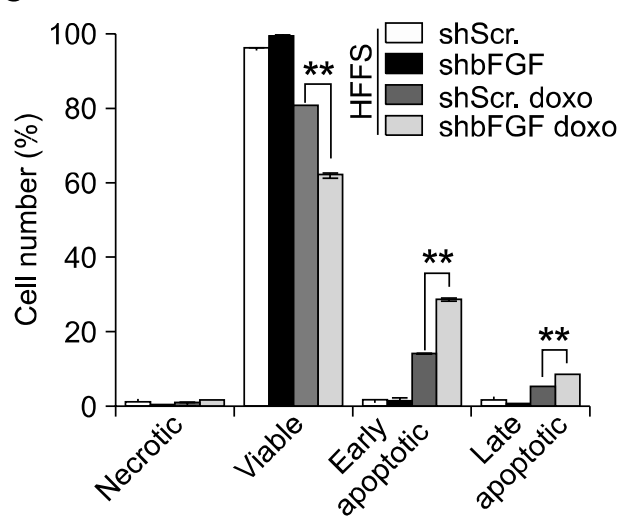

B
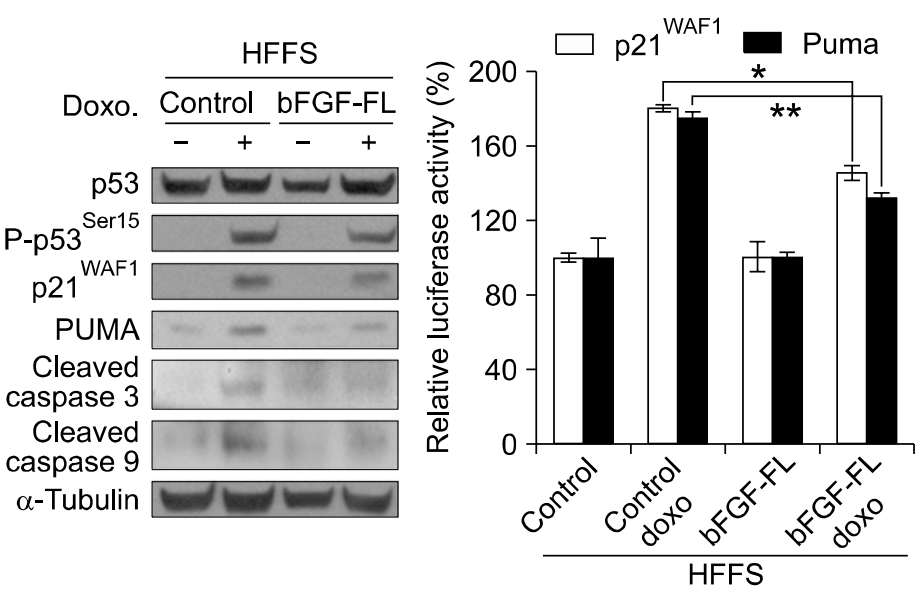

D

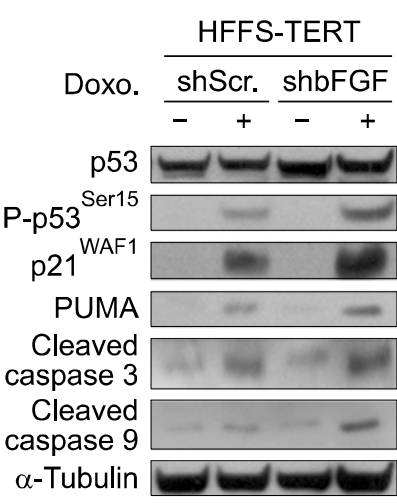

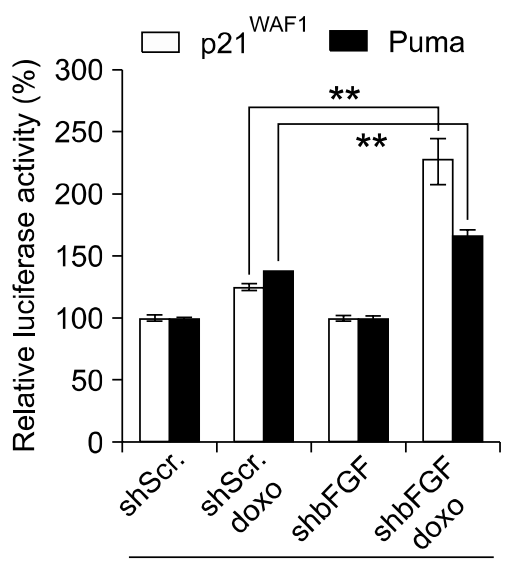

HFFS-TERT

Figure 3. bFGF induces resistance to DNA damage response and inactivation of p53 function. (A) Apoptotic cell population of HFFS-control and HFFS-bFGF full length (FL) is determined by AnnexinV-PI staining and FACS analysis. Doxorubicin (1 uM, $12 \mathrm{~h}$ ). (B) Expression levels of total p53, phosphorylated p53 (Ser15), p21 ${ }^{\text {WAF1 }}$, PUMA, cleaved caspase 3 and caspase 9 proteins in cells shown (A) are determined by Western blot analysis (left). Transcriptional activity of p53 was examined by measuring luciferase activity of p21 ${ }^{\text {WAF1 }}$-promoter- and PUMA-promoter-reporter vectors. (C) Apoptotic cell population of HFFS-TERT-shScramble and HFFS-TERT-shbFGF cells is determined by AnnexinV-PI staining and FACS analysis. Doxorubicin (1 uM, $12 \mathrm{~h}$ ). (D) Expression levels of total p53, phosphorylated p53 (Ser15), p21 ${ }^{\text {WAF1 }}$, PUMA, cleaved caspase 3 and caspase 9 proteins in cells shown (C) are determined by Western blot analysis (left). Transcriptional activity of p53 was examined by measuring luciferase activity of p21 ${ }^{\text {WAF1 }}$-promoter- and PUMA-promoter-reporter vectors.

\section{Role of TERT in the bFGF expression and cell proliferation}

Recently, ectopic expression of TERT in human mammary epithelial cells has been shown to accelerate cell proliferation and to change gene expression profiles (Smith et al., 2003). Especially, among a number of genes, such as EGFR and bFGF, that are upregulated in these cells, EGFR was found to be sufficient to stimulate cell proliferation, whereas the effect of bFGF on cell proliferation has not been studied. Therefore, we first examined whether bFGF is upregulated in HFFS-TERT cells. We found that bFGF expression was dramatically elevated in HFFS-TERT cells compared to HFFS-control cells (Figure 2A left).
Expression level of phosphorylated ERK1/2 a downstream effector of bFGF signaling in the HFFS-TERT cells was also increased at a similar level to that shown in the HFFS-control cells treated with bFGF (Figure 2A right). To determine whether increased expression of bFGF in the HFFS-TERT cells is required for telomerase activity, we transduced a telomerase activity-deficient TERT mutant (TERT-D712A) in HFFS cells. Consistent to previous reports (Harrington et al., 1997; Hahn et al., 1999; Zhang et al., 1999), ectopic expression of TERT-D712A mutant in HFFS cells did not induce an increase of telomerase activity, and in fact, telomerase activity in these cells show relatively decreased as compared 
A

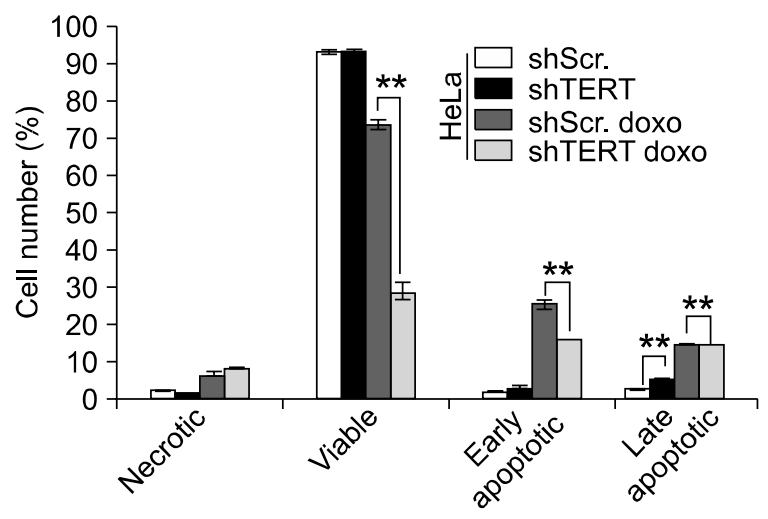

C

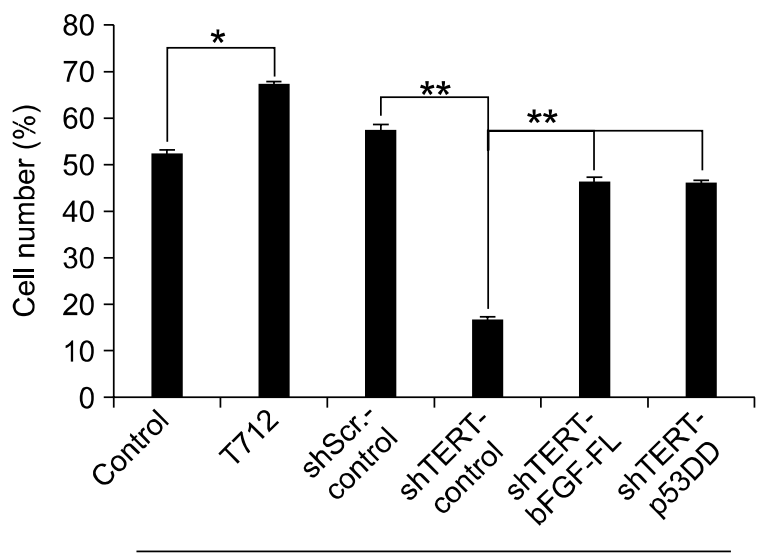

B

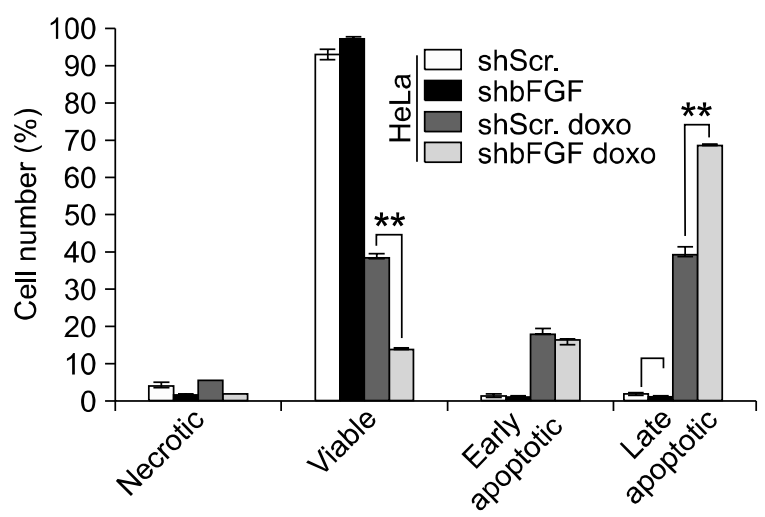

D

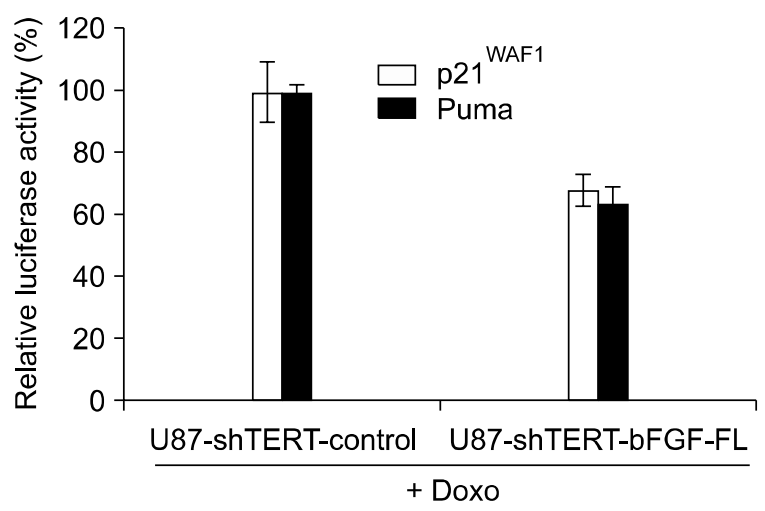

U87MG

Figure 4. Increases of DNA-damaging sensitivity as well as decrease of bFGF expression in cancer cells by depletion of TERT. (A) Apoptotic cell population of HeLa-shScramble and HeLa-shTERT cells is determined by AnnexinV-PI staining and FACS analysis. Doxorubicin (2 uM, $17 \mathrm{~h}$ ). (B) Apoptotic cell population of HeLa-shScramble and HeLa-shbFGF cells is determined by AnnexinV-PI staining and FACS analysis. Doxorubicin (2 uM, $17 \mathrm{~h})$. (C) Viable cell population of U87MG ectopically expressing TERT-D712A, shTERT, shTERT with bFGF-FL, and shTERT with p53DD were examined by AnnexinV-PI staining and FACS analysis. (D) Transcriptional activity of p53 in U87MG-shTERT-control and U87MG-shTERT+bFGF-FL cells grown with Doxorubicin $(2 \mathrm{uM}, 17 \mathrm{~h})$ was examined by measuring luciferase activity of p2 ${ }^{\text {WAF1 }}$-promoter- and PUMA-promoter-reporter vectors.

to parental HFFS-control cells (Figure 2B), possibly due to its dominant-negative effect against to wild-type TERT (Zhang et al., 1999). However, TERT-D712A mutant enabled to induce bFGF expression at a similar level to that shown in the HFFS-TERT (Figure 2B inset), which suggest that bFGF expression by TERT is independent of telomerase activity. Furthermore, similar to findings for HFFS-TERT cells, HFFS-TERT-D712A cells grown in the presence of doxorubicin are significantly more resistant to cell death compared to HFFS-control cells (Figure 2C). We next transduced full length bFGF into HFFS cells to determine whether ectopic expression of bFGF is able to induce cell proliferation, achieved by overexpression of TERT. Overexpression of bFGF in the HFFS cells leaded to significant increases in phosphorylated ERK $1 / 2$ and cell proliferation rate (Figure 2D), all which are quite comparable to those shown in the HFFS-TERT cells.

\section{Effect of bFGF on the DNA damage response and p53 function}

Since ectopic expression of TERT in HFFS cells showed resistance to DNA damage response and inactivation of p53 function, we examined whether bFGF in these cells also has similar functions to DNA damage response and p53 activity. We found that, like HFFS-TERT cells, HFFS-bFGF cells grown in the presence of doxorubicin are more resistant to cell death as compared to HFFS-control cells (Supplemental Data Figure S1B). Moreover, as determined by FACS analysis, the numbers of 
early and late apoptotic cells were significantly decreased, whereas necrotic cells were shown to be relatively increased in HFFS-bFGF cells as treated with doxorubicin (Figure $3 \mathrm{~A}$ ). We found that expression levels of phosphorylated p53 (Ser15), $\mathrm{p} 21^{\mathrm{WAF} 1}$, and Puma, and cleaved caspase 3 and caspase 9 proteins as well as transcriptional activity of p53 to p21 WAF1 and Puma promoters were significantly decreased in the HFFS-bFGF cells compared to HFFS-control cells, as treated with doxorubicin (Figure 3B).

Next, we transduced bFGF-shRNA into HFFSTERT cells to deplete expression of bFGF. Expression levels of bFGF mRNA and phosphorylated ERK1/2 proteins were markedly decreased in bFGFshRNA-transduced HFFS-TERT (HFFS-TERT-shbFGF) cells compared to control-shRNA vector-transduced HFFS-TERT (HFFS-TERT-shScramble) cells (Supplemental Data Figure S1C), indicating that bFGF-shRNA expression effectively suppresses its expression and signaling activity. We determined whether resistance to DNA damage response and inactivation of p53 function by TERT is achieved through overexpression of bFGF. As treated with doxorubicin and analyzed by FACS analysis, the number of viable cells were significantly decreased, whereas early and late apoptotic cells were dramatically increased in the HFFS-TERT-shbFGF cells compared to HFFS-TERT-shScramble cells (Figure $3 C)$. Furthermore, expression levels of phosphorylated p53 (Ser15), p21 ${ }^{\text {WAF1 }}$, and Puma, and cleaved caspase 3 and caspase 9 proteins as well as transcriptional activity of p53 to p21 WAF1 and Puma promoters were relatively increased in the HFFS-TERT-shbFGF cells compared to HFFSTERT-shScramble cells, as treated with doxorubicin (Figure 3D). Therefore, these results indicate that resistance to DNA damage response and inactivation of p53 function by TERT in the HFFS cells is achieved through increased bFGF expression.

\section{Effect of TERT depletion on cell death and p53 activity of HeLa and U87MG cells}

To determine whether the role of TERT in the DNA damage response is generally conserved in different cell types, we depleted the expression of TERT in HeLa cells using a TERT-specific shRNA expression. We found that the expression levels of bFGF mRNA and phosphorylated ERK1/2 proteins were dramatically decreased in HeLa-shTERT cells compared to HeLa-shScramble cells (Supplemental Data Figure S1D). Consistent to findings for HFFS cells, as treated with doxorubicin and analyzed by FACS analysis, the number of viable cells were significantly decreased, whereas the numbers of early and late apoptotic cells were dramatically increased in the HeLa-shTERT cell population compared to the HeLa-shScramble cell population (Figure 4A). Next, we transduced bFGF-shRNA into HeLa cells to deplete the expression of bFGF. Expression levels of bFGF mRNA and phosphorylated ERK1/2 proteins were markedly decreased in HeLa-shbFGF cells compared to HeLa-shScramble cells (data not shown). When treated with doxorubicin and analyzed by FACS analysis, the number of late apoptotic cells was dramatically increased in the HeLa-shbFGF cells compared to HeLa-shScramble cells (Figure $4 B$ ). The increased cell death observed in the HeLa cells by shRNA is recovered by exogenous bFGF treatment. The number of viable cells was significantly increased, whereas the numbers of necrotic and late apoptotic cells were markedly decreased in the bFGF-treated HeLa-shTERT cells compared to the HeLa-shScramble cells (data not shown). These results suggest that the sensitivity to the DNA damage response in HeLa-shTERT cells is partially increased due to the downregulation of bFGF in these cells. To determine tumorigenic activity that might be regulated by TERT and bFGF, we conducted an in vivo tumor formation assay by subcutaneous injection of HeLa-shScramble, HeLa-shTERT, and HeLa-shbFGF cells into nude mice. The size of tumors from HeLa-shTERT and HeLa-shbFGF was markedly decreased compared to those from HeLa-shScramble cells (Supplemental Data Figure S1E).

In addition, we also determined a crucial role of TERT and bFGF in the anti-apoptotic response using human U87MG glioma cell line that expresses endogenous TERT and wild-type p53. We have established U87MG cells exogenously expressing TERT-D712A, shTERT, shTERT+bFGF-FL, and shTERT+p53DD (a dominant-negative p53 mutant) (Supplemental data Figure S1F). As shown in Figure 4C, ectopic expression of TERT-D712A could enhance resistance to DNA damage-induced cell death. Conversely, repression of TERT with shRNA significantly attenuated DNA damage-mediated cell death in U87MG cells that were ectopically restored with bFGF and p53DD. We also found that transcriptional activity of p53 to p21 $21^{\text {WAF1 }}$ and Puma promoters were significantly decreased in the U87MG-shTERT+bFGF-FL cells compared to their counterpart cells, as treated with doxorubicin (Figure 4D). These results indicate that overexpression of bFGF and concomitant attenuation of p53 function by TERT should be a common mechanism in the different cell types. 


\section{Discussion}

A number of genetic alterations to escape a variety of cell death signals, such as a loss of p53 function that is involved in cell arrest and cell death under various cytotoxic stimuli (Vogelstein et al., 2000; Vousden and Lu, 2002; Perfettini et al., 2004), are one of the prerequisite events achieved by cancer cells. Regarding this, although telomerase showed inhibition of cell death through modulation of mitochondria-mediated apoptotic pathway (Massard et al., 2006), our findings here present that ectopic expression of TERT is capable of suppressing a classical p53-dependent cell death, which might be independent of activity of telomerase for telomere lengthening, because ectopic expression of telomerase activity-deficient mutant TERT (TERT-D712A) also markedly suppresses cell death induced by DNA-damaging agents. Although previous study showed that bFGF induces expression of MDM2 and its phosphorylation at serine 166, which are associated with inactivation of p53 function (Romanov et al., 2005), we found that ectopic expression of TERT induces upregulation of bFGF expression and p53 inactivation. Given that depletion of TERT or bFGF expression by RNA interference showed a marked elevation of phosphorylated p53 protein and its transcriptional activity for downstream-target genes (p21 WAF1 and Puma), the resistance to p53-dependent cell death by TERT is achieved by the TERT-mediated induction of bFGF expression. Similar to our findings, a previous study has demonstrated that depletion of insulin-like growth factor (IGF) receptor stimulates activation of ATM (Macaulay, 2004), which is one of the DNA damage sensors and activates $p 53$ function through induction of p53 phosporylation (Kurz and Lees-Miller, 2004). Since bFGF signaling, such PI3K/AKT and MEK/ERK, are mechanistically similar to IGF signaling (Laviola et al., 2007), the regulation of p53 activity through ATM may be also associated with TERT-mediated induction of bFGF expression. Therefore, determining how inactivation of p53 function by a TERT-bFGF regulatory axis will be challenging, and we hope to obtain insight into this question in the future.

\section{Methods}

\section{Cell culture and cell cycle analysis}

Immortalized human fetal fibroblasts (HFFS), normal human foreskin fibroblast (BJ), human cervical cancer cells (HeLa) and human glioma cells (U87MG) were maintained in Dulbecco's modified Eagle's medium (DMEM) high glucose medium enriched with $10 \%$ fetal bovine serum (FBS, Hyclone), 1\% penicillin and streptomycin (GibcoBRL), and $2 \mathrm{mM}$ L-glutamine (GibcoBRL). To determine cell growth rates, cells were plated at a density of $1 \times 10^{4}$ cells/6-well plate. Every two days after cell plating, cell numbers were counted using a hemacytometer. For cell cycle analysis, cells were fixed in cold $70 \%$ ethanol for at least $30 \mathrm{~min}$ at $-20^{\circ} \mathrm{C}$, washed twice with PBS, and then resuspended in $1 \mathrm{ml}$ of a propidium iodide solution $(250 \mathrm{ug} / \mathrm{ml}$ propidium iodide and $250 \mathrm{ug} / \mathrm{ml}$ RNase A) at $37^{\circ} \mathrm{C}$ for $30 \mathrm{~min}$. The fluorescent intensities $(10,000$ cells) were measured using a FACS Calibur (BD Biosciences). Forward scatter versus side light scatter and fluorescent area versus width were used to make a gate for intact single cells to evaluate for DNA content.

\section{Plasmid, shRNA construction, transfection, and retroviral infection}

HFFS cells were transfected with pCl-TERT-Neo $(4 \mu \mathrm{g})$ and pCDNA3.1-T712-Puro $(4 \mu \mathrm{g})$ using Lipofectamin 2000 (Invitrogen) according to the instructions of the manufacturer. Twenty four hours after transfection, cells were subjected to drug selection for 2 weeks. Cells were infected with retrovirus produced from the PT67 amphotropic packaging cell line (Clontech) transfected with retroviral vectors ( $p W G L-b F G F-B l a s t$, and $p B a b e-H-r^{2}{ }^{v 12}$-Puro). The cells plated $24 \mathrm{~h}$ earlier at $10^{6}$ cells $/ 10 \mathrm{~cm}$ dish were transduced by refeeding them with pre-filtered $(0.45 \mu \mathrm{m})$ retroviral supernatant containing $6 \mu \mathrm{g} / \mathrm{ml}$ of polybrene (Sigma). Cells were infected with retrovirus with bFGF-shRNA which were cloned into pSuperRetro-Puro (a constitutive RNAi expression vector), and TERT-shRNA which were cloned into PMKO-Puro (the constitutive RNAi expression vectors). The target sequences were human bFGF-shRNA: AAGCTACAACTTCAAGCAGAA; TERTshRNA: TTTCATCAGCAAGTTTGGA.

\section{Semi-quantitative reverse transcriptase-polymerase chain reaction (RT-PCR)}

Total RNA was isolated from cells using TRIzol (GibcoBRL) according to the instructions of the manufacturer. For semi-quantitative RT-PCR, $3 \mu \mathrm{g}$ of DNase I-treated RNA was converted to CDNA with Superscript II reverse transcriptase (Invitrogen) according to the instructions of the manufacturer. For semi-quantitative RT-PCR, a portion $(1 \mu \mathrm{l})$ of the RT reaction was used to amplify TERT, bFGF, $\beta$-actin, and GAPDH fragments using the corresponding gene-specific primer sets. Detailed forward $(F)$ and reverse (R) primer sequences, melting temperatures and cycles for RT-PCR are TERT (F: 5'-ACGTCTTCCTACGCTTCATGTG-3', R: 5'-TGAAGGTGAGACTGGCTCTGAT-3', 55 ${ }^{\circ} \mathrm{C}, 35$ cycles); bFGF (F: 5'-GTGTGTGCTAACCGTTACCT-3', R: 5'-GCTCTTAGCAGACATTGGAAG-3', $55^{\circ} \mathrm{C}, 35$ cycles): $\beta$-actin (F: 5'-TCGTGCGTGACATTAAGGAG-3', R: 5'-TGATCTCCTTCTGCATCCTG-3', $58^{\circ} \mathrm{C}, 25$ cycles), GAPDH (F: 5'-CTACACTGAGCACCAGGTGGTCTC-3', R: 5'-GATGGATACATGACAAGGTGCGGC- 3 ', $55^{\circ} \mathrm{C}, 25$ cycles). All semi-quantitative RT-PCR amplifications were verified to be in the linear range. Primers for real time-RT-PCR are 
TERT (F: 5'-CACGCGAAAACCTTCCTCA-3', R: 5'-CAAGTTCACCACGCAGCC-3', 60 ${ }^{\circ}$.); $18 \mathrm{~S}$ rRNA (F: 5'-TGCATGGCCGTTCTTAGTTG-3', R: 5'-AGTTAGCATGCCAGAGTCTCGTT-3', $\left.60^{\circ} \mathrm{C}\right)$.

\section{Western blot analysis}

Whole cell extracts prepared using RIPA lysis buffer were quantitated using the Bradford assay reagent (Bio-Rad) according to the instructions of the manufacturer. Protein in the extracts $(30-100 \mu \mathrm{g})$ was separated by a $4-12 \%$ gradient or $10 \%$ SDS-PAGE NuPAGE gel (Invitrogen) and transferred to PVDF membrane (Millipore). The membranes were blocked with $5 \%$ non-fat milk and incubated with anti-p53 (FL-393, Santa Cruz Biotechnology for normal p53 protein; OP03, Calbiochem for p53DD protein), anti-P-p53 $^{\text {Ser15 }}$ (16G8, Cell Signaling), anti-p21 WAF1 (C-19, Santa Cruz Biotechnology), anti-PUMA (ab-1, Calbiochem), anti-EGFR (1005, Santa Cruz Biotechnology), anti-ERK (Cell Signaling), anti-P-ERK (Cell Signaling), anti-actin (I-19, Santa Cruz Biotechnology), anti-cleaved caspase 3(Ab-2, Calbiochem), anti-cleaved caspase 9 (\#9502, Cell Signaling) and anti- $\alpha$-tubulin (Sigma) antibodies. Membranes were then incubated with horseradish peroxidase-conjugated anti-secondary IgG (Pierce) antibody and visualized with SuperSignal West Pico Chemiluminescent Substrate (Pierce).

\section{Subcutaneous implantations for tumorigenicity assay}

For subcutaneous implantation assay, cells $\left(1 \times 10^{6}\right)$ were subcutaneously transplanted into nude mice (BALB/c $\mathrm{nu} / \mathrm{nu}$ ). The mice were kept under observations during 2-3 months. All mouse experiments were performed in accordance with government and institutional guidelines and regulations.

\section{Luciferase-reporter gene activity assay}

p21 WAF1 and Puma transcriptional activity in the cells was determined by analyzing the relative luciferase acitivities of pGL3-p21 WAF1 - and pGL3-Puma-promoter plasmid using the Dual-Glo Luciferase Assay System (Promega). According to the instructions of the manufacturer (Promega), transfection efficiency was normalized with the activity of Renilla luciferase, which was cotransfected with the aforementioned pGL3-luciferase-reporter constructs.

\section{Assays for cytotoxicity and telomerase activity}

The trypan blue exclusion method was used to assess the viability and death rates of cells grown in the absence or presence of 1-2 $\mu \mathrm{M}$ doxorubicin for 12-17 $\mathrm{h}$. Cell death was determined by flow cytometry after cells were double-stained with Annexin V-FITC (a dilution of 1:100, BD PharMingen) and propidium iodide (50 ug/ml, Sigma). Cells were fractioned by viable cells (Annexin $\mathrm{V}^{+} / \mathrm{PI}^{-}$), early apoptotic cells (Annexin $\mathrm{V}^{-} / \mathrm{Pl}^{-}$), late apoptotic cells (Annexin $\mathrm{V}^{+} / \mathrm{PI}^{+}$) and necrotic cells (Annexin $\mathrm{V}^{-} / \mathrm{PI}^{+}$). The percentage of cells was determined by flow cytometry using a FACS Calibur equipped with CellQuestPro software. Telomerase activity was assayed using the TRAPeze ELISA Detection Kit (Chemicon International, Inc., Temecula, CA) according to the instructions of the manufacturer.

\section{Statistics}

Data were analyzed statistically using a two-tail Student's $t$ test. The level of statistical significance stated in the text was based on the $P$ values. $P<0.05^{*}$ or $P<0.01^{* *}$ was considered statistically significant.

\section{Supplemental data}

Supplemental Data include a figure and can be found with this article online at http://e-emm.or.kr/article/article_files/ SP-42-8-05.pdf.

\section{Acknowledgements}

This work was supported by the National Research Foundation of Korea (NRF) grant funded by the Korea government (MEST) (2008-0058785) and Korea University Research Grant. X.J. was supported by a BK21 Research Fellowship from the Ministry of Education and Human Resources Development. S.H.K was supported by the National Research Foundation of Korea Grant funded by the Korean Government [NRF-2009-351-C00137].

\section{References}

Aylon Y, Oren M. Living with p53, Dying of p53. Cell 2007;130: 597-600

Branca M, Giorgi C, Ciotti M, Santini D, Di Bonito L, Costa S, Benedetto A, Bonifacio D, Di Bonito P, Paba P, Accardi L, Mariani L, Ruutu M, Syrjänen S, Favalli C, Syrjänen K. Upregulation of telomerase (TERT) is related to the grade of cervical intraepithelial neoplasia, but is not an independent predictor of high-risk human papillomavirus, virus persistence, or disease outcome in cervical cancer. Diag Cytopathol 2007;34:739-48

Cayuela ML, Flores JM, Blasco MA. The telomerase RNA component Terc is required for the tumour-promoting effects of Tert overexpression. EMBO J 2005;6:268-74

Gorbunova V, Seluanov A, Pereira-Smith OM. Expression of human telomerase (TERT) does not prevent stress-induced senescence in normal human fibroblasts but protects the cells from stress-induced apoptosis and necrosis. J Biol Chem 2002;277:38540-9

Greider CW. Telomere length regulation. Annu Rev Biochem 1996;65:337-65

Hahn WC, Stewart SA, Brooks MW, York SG, Eaton E, Kurachi A, Beijersbergen RL, Knoll JH, Meyerson M, Weinberg RA. Inhibition of telomerase limits the growth of human cancer cells. Nat Med 1999;5:1164-70

Harrington L, Zhou W, McPhail T, Oulton R, Yeung DS, Mar 
$\mathrm{V}$, Bass MB, Robinson MO. Human telomerase contains evolutionarily conserved catalytic and structural subunits. Genes Dev 1997;11:3109-15

Hiyama E, Hiyama K. Clinical utility of telomerase in cancer. Oncogene 2002;21:643-9

Kurvinen K, Syrjänen S, Johansson B. Long-term suppression of telomerase expression in HeLa cell clones, transfected with an expression vector carrying siRNA targeting TERT mRNA. Int J Oncol 2006;29:279-88

Kurz EU, Lees-Miller SP. DNA damage-induced activation of ATM and ATM-dependent signaling pathways. DNA Repair (Amst) 2004;3:889-900

Laviola L, Natalicchio A, Giorgino F. The IGF-I signaling pathway. Curr Pharm Des 2007;13:663-9

Lee MK, Hande MP, Sabapathy K. Ectopic mTERT expression in mouse embryonic stem cells does not affect differentiation but confers resistance to differentiation- and stress-induced p53-dependent apoptosis. J Cell Sci 2005; 118:819-29

Li S, Crothers J, Haqq CM, Blackburn EH. Cellular and gene expression responses involved in the rapid growth inhibition of Human cancer cells by RNA interference-mediated depletion of telomerase RNA. J Biol Chem 2005;280: 23709-17

Luiten RM, Pene J, Yssel H, Spits H. Ectopic TERT expression extends the life span of human CD4+ helper and regulatory $\mathrm{T}$-cell clones and confers resistance to oxidative stress-induced apoptosis. Blood 2003;101:4512-9

Macaulay VM. The IGF receptor as anticancer treatment target. Novartis Found Symp 2004;262:235-43

Massard C, Zermati Y, Pauleau AL, Larochette N, Metivier D, Sabatier L, Kroemer G, Soria JC. TERT: a novel endogenous inhibitor of the mitochondrial cell death pathway. Oncogene 2006;25:4505-14

Papadopoulou A, Trangas T, Teixeira MR, Heim S, Dimitriadis $\mathrm{E}$, Tsarouha $\mathrm{H}$, Andersen JA. Evangelou E, loannidis $P$. Telomerase activity and genetic alterations in primary breast carcinomas. Neoplasia 2003;5:170-8

Perfettini JL, Kroemer RT, Kroemer G. Fatal liaisons of p53 with Bax and Bak. Nat Cell Biol 2004;6:386-8

Romanov VV, James $\mathrm{CH}$, Sherrington PD, Pettitt AR. Basic fibroblast growth factor suppresses p53 activation in the neoplastic cells of a proportion of patients with chronic lymphocytic leukaemia. Oncogene 2005;24:6855-60

Rudolph KL, Millard M, Bosenberg MW. DePinho RA. Telomere dysfunction and evolution of intestinal carcinoma in mice and humans. Nat Genet 2001;28:155-9

Shay JW, Bacchetti SA. Survey of telomerase activity in human cancer. Eur J Cancer 1997;33:787-91

Smith LL, Coller HA, Roberts JM. Telomerase modulates expression of growth-controlling genes and enhances cell proliferation. Nat Cell Biol 2003;5:474-9

Tomoda R, Seto M, Tsumuki H, lida K, Yamazaki T, Sonoda $\mathrm{J}$, Matsumine A, Uchida A. Telomerase activity and human telomerase reverse transcriptase mRNA expression are correlated with clinical aggressiveness in soft tissue tumors. Cancer 2002;95:1127-33

Vogelstein B, Lane D, Levine AJ. Surfing the p53 network. Nature 2000;408:307-10

Vousden KH, Lu X. Live or let die: the cell's response to p53. Nat Rev Cancer 2002;2:594-604

Wang R, Lin F, Wang X, Gao P, Dong K, Wei SH, Cheng SY, Zhang $\mathrm{HZ}$. The therapeutic potential of survivin promoter-driven siRNA on suppressing tumor growth and enhancing radiosensitivity of human cervical carcinoma cells via downregulating hTERT gene expression. Cancer Biol Ther 2007;6:1295-301

Xu T, Rao Y, Zhu W, Guo F. Inhibition of cell growth and telomerase activity in osteosarcoma cells by DN-TERT. J Huazhong Univ Sci Technolog Med Sci 2006;26:601-3

Zhang X, Mar V, Zhou W, Harrington L, Robinson MO. Telomere shorting and apoptosis in telomerase-inhibited human tumor cells. Genes Dev 1999;13:2388-99 